\title{
DIGITALCOMMONS
}

@WAYNESTATE-

Wayne State University

Civil and Environmental Engineering Faculty

Research Publications

Civil and Environmental Engineering

$10-31-2020$

\section{Failure Sampling with Optimized Ensemble Approach for the Structural Reliability Analysis of Complex Problems}

\author{
Christopher Eamon \\ Wayne State University, eamon@eng.wayne.edu \\ Kapil Patki \\ J3 Engineering Group, kapil.patki@wayne.edu \\ Ahmad Alsendi \\ Wayne State University, ahmad.alsendi@wayne.edu
}

Follow this and additional works at: https://digitalcommons.wayne.edu/ce_eng_frp

Part of the Civil Engineering Commons, and the Structural Engineering Commons

\section{Recommended Citation}

Eamon, C., Patki, K., and Alsendi, A. 2021. "Failure sampling with optimized ensemble approach for the structural reliability analysis of complex problems." J. Risk Uncertainty Eng. Syst., Part A: Civ. Eng. 7 (1): 04020050. https://doi.org/10.1061/AJRUA6.0001100.

This Article is brought to you for free and open access by the Civil and Environmental Engineering at DigitalCommons@WayneState. It has been accepted for inclusion in Civil and Environmental Engineering Faculty Research Publications by an authorized administrator of DigitalCommons@WayneState. 
1 Failure Sampling with Optimized Ensemble Approach for the Structural Reliability Analysis of Complex Problems

\section{Abstract}

5 Failure sampling is a structural reliability method based on modified conditional expectation

6 suitable for complex problems for which reliability index-based approaches are inapplicable

7 and simulation is needed. Such problems tend to have non-smooth limit state boundaries or

8 are otherwise highly nonlinear. Previous studies recommended implementation of failure

9 sampling with an extrapolation technique using Johnson's distribution or the generalized

10 lambda distribution. However, what implementation method works best is problem dependent.

11 The uncertainty of which approach provides best results for a particular problem limits the potential effectiveness of the method. In this study, a solution is proposed to this issue that eliminates this uncertainty. The proposed approach is an optimized ensemble that forms a uniquely-weighted solution by utilizing the predictive capability of multiple curves to maximize accuracy for any particular problem. It was found that the proposed approach produces solutions superior to the methods of implementing failure sampling previously presented in the literature.

Keywords: numerical methods; optimization; uncertainty analysis; reliability analysis

1. Associate Professor of Civil and Environmental Engineering, Wayne State University, Detroit, MI 48202; eamon@eng.wayne.edu

2. Project Engineer, J3 Engineering Group; kapil.patki@wayne.edu.

3. Graduate student, Civil and Environmental Engineering, Wayne State University, Detroit, MI 48202; ahmad.alsendi@wayne.edu 
Introduction

A large number of structural reliability analysis methods have been proposed in the last several decades. The most common among these can perhaps be grouped into two broad divisions: simulation methods such as Monte Carlo Simulation (MCS) and its many variants; and analytical approaches that compute reliability index as a surrogate for direct evaluation of failure probability. This latter group of methods includes the ubiquitous First Order Reliability Method (FORM), which has become very popular since its introduction in the late 1970s with an adjustment for non-normal random variables (Rackwitz and Fiessler 1978). Although not as frequently used, Second Order Reliability Methods (SORM) have also been proposed (Breitung 1984), as well as similar reliability index based algorithms. Such methods attempt to locate the most probable point of failure (MPP), the peak of the joint probability density function on the failure boundary of the limit state function in standard normal space. Reliability index $(\beta)$ is then typically calculated as the distance from the MPP to the origin, from which a simple transformation to failure probability can be obtained. Although computationally efficient, usually offering vast reductions of computational effort for typical problems over simulation approaches, reliability index based methods cannot guarantee convergence to the true solution, unlike MCS if the sample size is sufficiently increased. Rather, as reliability index methods rely upon an approximation of the limit state boundary at the MPP (a linear approximation in the case of FORM), nonlinearities in standard normal space in which $\beta$ is calculated, either from inherent nonlinearities in the structural response considered for the limit state function, or when non-normal random variables are introduced, will result in some degree of error. Although this error is often small, in some cases, particularly for complex nonlinear limit state functions, it can be unacceptably large (Eamon et al. 2005; Melchers 1999; Chiralaksanakul and Mahadevan 2005; Haldar and Mahadevan 2000). For other complex 
MPPs, search algorithms are sometimes unable to identify the MPP, and the solution process fails completely (Patki and Eamon 2016; Eamon and Charumas 2011).

Although direct simulation such as MCS may approach the true solution as sample size is increased, the well-known drawback of such methods is the large computational demand for complex engineering problems, particularly such as those requiring finite element analysis for solution. To increase efficiency, numerous variance reduction modifications were proposed to MCS, including stratified sampling (Iman and Conover 1982), importance sampling (Rubinstein 1981; Engelund and Rackwitz 1993), adaptive importance sampling (Wu 1992; Karamchandani et al. 1989), directional simulation (Ditlevesen and Bjerager 1988), dimensional reduction and integration (Acar et al. 2010); subset simulation (Au et al. 2007), and many others. As with any approach, each of these methods has particular disadvantages. For example, stratified sampling, of which perhaps Latin Hypercube (Iman and Conover 1982) is among the most well-known, has not consistently shown significant reductions in computational costs for a variety of problems (Eamon et al. 2005). Importance sampling, as with reliability index methods, which require identification of the MPP, may obtain inaccurate or no solution for complex problems. Although directional simulation is extremely efficient for some problems, particularly for limit state boundaries that are spherical, efficiency is reduced when the limit state boundary takes on a common hyperplanar shape (Engelund and Rackwitz 1993). More recent advancements, however, such as adaptive directional importance sampling, have maintained high efficiency for a variety of non-spherical limit state boundaries when the MPP can be located (Grooteman 2011; Shayanfar 2018). Subset simulation has been developed significantly in the last two decades, producing various alternative implementation approaches. An important consideration with this method, however, is how the importance sampling density is determined, as high variance in the solution can be obtained with suboptimal selections (Au and Beck 2001; Au et al. 2007). 

itself can be simplified. One systematic way to achieve this is with use of a response surface, where a computationally demanding limit state function evaluation can be replaced with a more simple, analytical surrogate function (Gomes and Awruch 2004; Cheng and Li 2009). The response surface can then be used with a variety of traditional reliability analysis methods to provide fast probabilistic solutions. Alternative surrogate models include those developed from polynomial chaos expansion, Kriging, genetic algorithms, and artificial neural networks (ANN), among others (Gomes 2019; Guo et al. 2020). Adaptive versions of such metamodeling techniques, particularly ANN, have shown to be effective for solving a variety of complex problems (Gomes 2019). However, in some cases, the cost of forming a highfidelity surrogate model for a complex problem may outweigh the cost of using the original response with a reasonably efficient reliability method to begin with (Eamon and Charumas 2011).

As an alternative solution for complex reliability problems, Eamon and Charumas (2011) proposed the modified conditional expectation, or failure sampling (FS) method, which was reported to accurately solve various complex limit states with reasonable computational effort. In general, the method uses conditional expectation to sample the complex (generally resistance) portion of the limit state function, then uses a numerical technique to estimate either its probability density function (PDF) or cumulative distribution function (CDF). Failure probability can then computed directly by numerical integration over the failure region.

100 Alternatively, additional resistance data for high reliability problems can be generated by extrapolation, where the original sample is fit to a flexible, multi-parameter curve to extend the tail region. Clearly, the accuracy of this approach is a function of how well the PDF or CDF estimate and resulting curve fit are developed. As might be expected, what implementation method works best is problem dependent. Unfortunately, there is little a priori indication as to 
what method produces the greatest accuracy for a specific problem. For example, Patki and Eamon (2016) examined various problems with the FS approach and generally recommended data extrapolation using Johnson's Distribution, as they found it produced best results in many cases. However, this is not always true, as using alternative flexible curves such as the generalized lambda distribution or the generalized extreme value distribution produced higher accuracy for some problems. The uncertainty of which approach provides best results for a particular problem limits the potential effectiveness of the method. This issue is the focus of this study. Here, an optimized ensemble approach is developed, with minimal additional computational effort, that forms a uniquely-weighted fit by utilizing the predictive capability of multiple curves that maximizes the accuracy of the FS method for any particular problem.

\section{Summary of Failure Sampling Method}

The FS approach is fully described elsewhere (Eamon and Charumas 2011; Patki and

118 Eamon 2016), whereas a brief summary is provided here. The probability of failure $p_{f}$ of a

119 limit state function $g$ can be calculated by estimating a single-dimensional PDF of $g$ and integrating the PDF over the failure region (i.e. where $\mathrm{g}<0$ ). Direct MCS can be used to generate the sample of $g$ used to develop the PDF. Of course, this approach will yield accurate results only when the PDF of $g$ can be estimated accurately. However, for typical structural reliability problems, the large majority of the sample generated from MCS is far from the

124 failure region, resulting in a problem for which it is difficult if not impossible to accurately integrate the failure region without a high number of simulations.

In the FS approach, the initial limit state function $g\left(\boldsymbol{X}_{i}\right)$, consisting of random variables

$127 \boldsymbol{X}_{\boldsymbol{i}}$, is reformulated to a new limit state $g^{*} . g^{*}$ is expressed in terms of a control random variable

$128 Q$, and the function of remaining random variables $(\mathrm{RVs}), R\left(\boldsymbol{X}_{\boldsymbol{j}}\right)$. Setting $g *$ to zero to represent

129 the failure boundary, the problem can be written as: 
131 Here $g *$ is mathematically equivalent to original limit state function $g$. Note that function $R\left(\boldsymbol{X}_{j}\right)$

132 need not be explicitly formed and could be evaluated from FEA or another complex numerical

133 procedure. Moreover, there is no theoretical limitation in the selection of $Q$, although it is often

134 chosen as a load RV in the physical problem for convenience of implementation. For greatest accuracy, it is best that $Q$ is selected such that it is statistically independent of the remaining $\mathrm{RVs} \boldsymbol{X}_{j}$, a stipulation that can be satisfied for at least one RV by nearly all realistic structural reliability problems. Further, it is advantageous to select this variable as that with the highest variance, if possible, which removes its associated uncertainty from the simulated data and may reduce the number of simulations required for the same accuracy. If multiple RVs exist with the same variance, there is no theoretical advantage of selecting one over another as the control variable, and the choice reduces to that of convenience. Once Eq. 1. is formed, values $\boldsymbol{x}_{\boldsymbol{j}}$ are simulated by a method such as MCS. From Eq. 1., it can be seen that for a particular set of 143 simulated values $R\left(x_{j}\right), q=R\left(x_{j}\right)$. That is, if a value $q$ can be determined to satisfy Eq. 1, that 144 value also equals a datum for the sample of $R\left(x_{j}\right)$. Note for complex problems, this generally requires a non-linear solver to determine $q$, as further discussed in a detailed summary of the procedure given below. A value $q$ is thus determined for each set of simulated values $R\left(\boldsymbol{x}_{j}\right)$, thereby developing an equivalent, single-dimensional data sample for the potentially very complex, multi-variate $R\left(\boldsymbol{X}_{j}\right)$. Once the data sample for $R\left(\boldsymbol{X}_{j}\right)$ is generated, there is no need to evaluate the true response further (e.g. no need for further FEA, if that is how the limit state

150 function is evaluated), and the bulk of the computational effort for a complex problem ends.

151 Next, depending on the solution approach, a PDF or CDF estimate of $R\left(\boldsymbol{X}_{j}\right)$ is developed, from which $p_{f}$ of $g *$ (and thus of the original limit state function $g\left(\boldsymbol{X}_{\boldsymbol{i}}\right)$ ) can then be found with a variety of methods. As mentioned above, one approach is numerically integrating over the region with: 


$$
p_{f}=\int_{-\infty}^{\infty} F_{R}(q) f_{Q}(q) d q
$$

156 where $F_{R}$ refers to the CDF of $R$ and $f_{Q}$ the PDF of $Q$. Due to the sparsity of data with high157 reliability problems, numerical integration may lose accuracy. Thus, an alternative approach

158 is to use a flexible curve to represent the data sample for $R\left(X_{j}\right)$, which can be used to extend 159 the tail region indefinitely. Once this is done, $p_{f}$ can be computed very quickly with any method, 160 such as MCS, for example, as the original, potentially complex function $g *$ is now represented analytically.

In summary, the FS approach offers several useful features:1) as the MPP is not used, problems for which the MPP cannot be located, or which is incorrectly located, and thus which are unsolvable or inaccurately solved by reliability index or importance sampling methods, can be addressed; 2) for complex problems of moderate reliability (i.e., reliability index from about 3-5, within the range of typical structural components) that are poorly solved with many other methods, computational effort for FS is relatively low, often on the order of 1000 simulations, for reasonably accurate solutions; 3 ) the method is simple conceptually as well as to implement. As suggested above, as a simulation-based method, although FS is applicable to any type of

170 problem, it becomes competitive when reliability-index based methods provide no or poor 171 solutions, and for which other simulation methods require an unfeasibly large computational effort. For simpler problem types, reliability index based solutions are generally more efficient.

173 Although the FS approach was previously demonstrated to be effective, as noted above, an existing concern is that it is not clear what method of implementation works best for different problem types. This is the issue that this study attempts to address.

Optimized Ensemble Approach 
combination of the multiple available possibilities. Ensembles have used to increase the accuracy of various problems, most notably in metamodeling (Zerpa et al. 2005; Goel et al. 2007; Acar and Rais-Rohani 2009; among others), where in much of this previous work, an ensemble of metamodels is generally represented as a weighted sum of two or more stand-

184 alone metamodels, each separately fitted to the same response using different techniques. The resulting hybrid metamodel takes advantage of the prediction ability of each individual standalone metamodel to enhance the accuracy of response predictions. CDFs of the resistance sample for $R\left(X_{j}\right)$ is established, then the individual CDFs are assigned weight factors depending upon their anticipated accuracy. Using a weighted sum formulation, a unique, problem-specific ensemble of CDFs can thus be formulated as follows:

$$
F_{R E}=\sum_{i=1}^{N} w_{i} F_{R T i}
$$

where $F_{R E}$ is the final ensemble CDF of $N$ stand-alone CDFs $F_{R T i}$, and $w_{i}$ is the weight factor of $i t h$ stand-alone CDF. In this study, three stand-alone CDFs are used, individually fit to the sampled data $R\left(\boldsymbol{x}_{j}\right)$ as discussed in more detail below. The weight factors are subjected to the following constraint:

$$
\sum_{i=1}^{N} w_{i}=1
$$

197 The weight factors are determined by a sequential quadratic programming optimization process

198 where the difference between the CDF formed directly from the sampled data, the "true" CDF, 199 and the analytical representation, $F_{R E}$, is minimized. The CDF of the sampled data of $M$ total 200 points can be expressed as:

$$
F_{R}(s)=\frac{s}{1+M}
$$


where $F_{R}(s)$ is the CDF value for datum $s$. Although numerous goodness-of-fit metrics exist, the error between the true $\mathrm{CDF}$ and $F_{R E}$ is measured in this study using a generalized mean square error (MSE) metric given as:

$$
M S E=\frac{1}{M} \sum_{s=1}^{M}\left(F_{R E}(s)-F_{R}(s)\right)^{2}
$$

The final optimization problem is to find the optimal values of design variables $\boldsymbol{W}=\left(w_{1}, w_{2}\right.$, $\left.\ldots ., w_{N}\right)$ that would

$$
\min \quad \operatorname{Err}=\mathrm{MSE}=\mathrm{f}(\boldsymbol{W})
$$

$$
\text { s.t. } \quad \sum_{i=1}^{N} w_{i}=1
$$

$$
0 \leq w_{i} \leq 1
$$

211 Prior to recommending MSE, the authors studied two alternative forms of goodness-of-fit: a

212 log-based criterion to maximize differences in the lower resistance tail, similar to the Anderson-

213 Darling test (Ang and Tang 2007); as well as that based on the linear sum of differences in 214 CDFs, as per Eamon and Charumas (2011). It was found that both could produce better results than MSE in some cases, but significantly worse in other cases, and for problems with no apparently similar characteristics. As opposed to the alternative metrics, which were less

217 reliable overall, it was found that MSE tends to emphasize differences in weights, focusing the 218 weighted response on a single dominant distribution.

As three stand alone CDFs were used in this study, at the start of the optimization, the initial values for the weight factors $\boldsymbol{W}$ are set to $1 / 3$. Once a data sample for $R\left(X_{j}\right)$ is generated,

221 the alternate CDFs are individually fit to the data and the weights are determined. A realized 222 value for $R\left(X_{j}\right), r$, is then represented as the weighted sum of the values $r_{i}$ taken from the stand 223 alone CDFs, as determined in accordance with Eq. 3, which results in: 


$$
r=\sum_{i=1}^{N} w_{i} r_{i}
$$

225 As with the original FS method, any reliability method such as MCS or FORM can then be 226 used to quickly determine the $p_{f}$ estimate of $g^{*}$, since $R\left(\boldsymbol{X}_{j}\right)$ is now represented as a fully 227 defined, single dimensional random variable $R$. For example, if MCS were used to solve Eq. 2281 , a realized value $r$ is determined by sampling each of the stand-alone CDFs (using the same random perturbation for each curve per simulation) to produce $N$ values, which are then combined in accordance with Eq. 8. Note that, for a complex problem, it is the generation of the data sample for $R\left(X_{j}\right)$ that requires calling the original limit state function (assumed to be a time-consuming FEA code or similar analysis tool), where the complex, multidimensional $R\left(X_{j}\right)$ is transformed to an equivalent single RV via the original FS process. Once the data sample for $R\left(\boldsymbol{X}_{j}\right)$ is established, the computational effort required for all further calculations, including the developments of the alternative CDFs and their weight factors, as well as to estimate $p_{f}$ of $g *$, is comparatively trivial (generally seconds of real time on a modern desktop computer). In this study, after the data sample for $R\left(X_{j}\right)$ was established and the ensemble CDF weights determined to complete Eq. 3, MCS was used in conjunction with Eq. 8 to compute $p_{f}$ of $g^{*}$. The procedure can be summarized as follows:

The original limit state function is rewritten in the form of Eq. 1.

1. Values for $\mathrm{RVs}$ within $R\left(\boldsymbol{X}_{\boldsymbol{j}}\right)$ are determined by simulation. In this study, direct MCS is used, although other techniques are also possible (Patki and Eamon 2014). A single realized value $R\left(x_{j}\right)$ is thus determined.

2. The required value for $Q$ necessary to satisfy Eq. 1 is determined. For a simple problem for which the limit state can be explicitly written, $q$ can readily be found to be given by $R\left(\boldsymbol{x}_{j}\right)$, as from Eq. $1, R\left(\boldsymbol{x}_{j}\right)=q$. For more complex problems, where Eq. 1 may be implicit, a nonlinear solver is required to determine $q$. An example 
of this scenario is for problems that involve a finite element procedure, where the relationship between the measured response in the limit state function (for example, stress or displacement) and the value of $q$, as a function of values for the remaining variables $\boldsymbol{x}_{j}$, cannot be explicitly established. In practice, Eq. 1 would then be solved by incrementing the value of $q$ until the specified failure criterion (such as a stress or displacement limit) is achieved.

3. The simulation process is repeated (i.e. steps 2 and 3) until the desired sample size is generated. As with most simulation methods, increasing the sample size generally improves results. However, in this study, 1000 simulations were used. At the conclusion of this step, the actual limit state function (which may be computationally expensive to evaluate) is no longer used in the problem solution.

4. Since $q=r$ on the failure boundary, the values determined for $Q$ also must equal corresponding values for $R\left(\boldsymbol{X}_{j}\right)$; conceptually, values of resistance. Independent curves (CDFs) are then fit to the (1000 point) data sample for $R\left(X_{j}\right)$, essentially reducing the potentially complex resistance function into a representative single random variate (albeit at this point, represented by a set of $i$ alternative CDFs). These CDFs are the individual member functions $\left(F_{R T i}\right)$ of the ensemble.

5. Using Eqs. 3-7, weights $w_{i}$ are assigned to each CDF by optimization. The final optimized CDF of resistance is then represented by Eq. 3.

6. Assuming that $Q$ is an $\mathrm{RV}$ with known parameters, Eq. 1. can now be explicitly expressed as: $g^{*}=R-Q$. This simple, two RV limit state function can be solved with any reliability method such as MCS, importance sampling, FORM, SORM, etc, as desired. Here, note that regardless of the method used, $i$ different values (i.e. the number of curves used to construct the ensemble) for RV $R$ are required for solution. For example, if using a simulation approach such as MCS, for one 
simulation, the same initially generated uniform random value would be used to resolve each of the alternative values $r_{i}$ from CDFs $F_{R T i}$. The final realized value $r$ is then given by the weighted sum of the realizations, per Eq. 8 .

To simplify the approach further, it was observed that in many problems a single CDF case, good results can often be obtained by simply using the single dominant curve, forgoing the ensemble. Thus, an effective simplified approach to determine values for $R$ can be implemented as follows:

$$
R=R_{T}, w_{T} \geq T h
$$

$$
R=E n s, \quad w_{T}<T h
$$

where $R_{T}$ is a value of $R$ determined from the single dominant distribution; Ens is the ensemble approach, given by Eq. $8 ; w_{T}$ is the weight of the dominant distribution found from the minimization of MSE per Eqs. 6 and 7; and Th is the dominant weight threshold to forgo the ensemble. The choice of $T h$ is subjective, representing a desired balance between accuracy and additional complexity, and is best left to the analyst. However, for many problems the authors have found good results for threshold weights as low as $T h=3 / 4$, as will be discussed below.

In general, the ensemble approach is intended for problems for which the MPP cannot

290 be located or the failure boundary is not well represented with a FORM/SORM approximation, and direct simulation methods are too costly. Limitations of the ensemble approach include the need for statistical independence of the control variable; the need for a nonlinear solver to set $g^{*}=0$ for implicit problems; and most importantly, the need for the surrogate distribution to accurately represent the CDF of $R$. Although seemingly challenging, the latter condition may be satisfied even for relatively complex problems, provided that some prior knowledge of the response is available. For example, if, say, a unique limit state boundary exists such as a truncated or multimodal form, then such a distribution type may be included within the 
ensemble, using one or more of the numerous representations of such distributions available in the literature. Alternatively, if only one troublesome distribution type is present, it may be taken as the control variable, removing it from the response to be fit.

Another issue to consider is that, as with any approximate approach, there is no guaranteed method to obtain the error associated with the reliability estimate, which would require knowledge of the true solution beforehand. However, as with most MCS-based approaches, it has been shown that increasing the number of simulations using the FS method produces estimates that tend to converge to the true solution (Eamon and Charumas 2011). Thus, although it is not possible to directly quantify the error associated with the proposed approach, it can at least be ensured that the number of simulations used is sufficient by increasing this number until subsequent results do not significantly differ.

\section{CDFs Considered}

In this study, CDFs are generated from the $R\left(x_{j}\right)$ data by three methods for use in Eq. 3: use

312 of the generalized lambda distribution (GLD), Johnson's distribution (JSD), and the 313 generalized extreme value distribution (GEV). Although any type and number of CDFs can 314 be included in the analysis, the approach taken here is the use of a smaller number of highly 315 flexible functions.

The GLD can represent many common distributions such as normal, lognormal,

317 Weibull, and others. It is defined by location $\left(\lambda_{1}\right)$, scale $\left(\lambda_{2}\right)$, skewness $\left(\lambda_{3}\right)$, and kurtosis $\left(\lambda_{4}\right)$

318 parameters. Various ways have been developed to estimate these parameters (Karian and 319 Dudewicz 2011; Ozaturk and Dale; Asif and Helmut 2000). The method of moments was used

$$
f_{R T}(x)=\frac{\lambda_{2}}{\left[\lambda_{3} u^{\left(\lambda_{3}-1\right)}+\lambda_{4}(1-u)^{\left(\lambda_{4}-1\right)}\right]}
$$



variable $x$ through the inverse of the $\mathrm{CDF}$, the quantile function: $x=Q(u)=\lambda_{1}+$ $324 \frac{u^{\lambda_{3}}-(1-u)^{\lambda_{4}}}{\lambda_{2}}$.

The Johnson's system of distributions is also defined by four parameters and similarly has wide flexibility. This system is composed of multiple normalizing transformations: the bounded, or ' $\mathrm{S}_{\mathrm{B}}$ ' transformation, which models distributions bounded on either or both ends such as gamma and beta; the semi-bounded ' $\mathrm{S}_{\mathrm{L}}$ ' transformation, which models a lognormal distribution, and the unbounded ' $\mathrm{S}_{U}$ ' transformation which can model the normal, $\mathrm{t}$, and other distributions. A JSD distribution is defined with two shape parameters $\gamma$ and $\delta$, a location parameter $\xi$, and a scale parameter $\lambda_{j}$. The Johnson's PDF is given by:

$$
f_{R T}(x)=\frac{\delta}{\lambda_{j} \sqrt{2 \pi}} g_{r}^{\prime}\left(\frac{x-\xi}{\lambda_{j}}\right) \exp \left[-\frac{1}{2}\left(\gamma+\delta g_{r}\left(\frac{x-\xi}{\lambda_{j}}\right)\right)^{2}\right]
$$
where function $g_{r}\left(\frac{x-\xi}{\lambda_{j}}\right)$ is determined by the desired transformation (i.e. either $\mathrm{S}_{\mathrm{B}}$, $\mathrm{S}_{\mathrm{L}}$, or $\mathrm{S}_{\mathrm{U}}$ ). As with the GLD, multiple methods are available to determine JSD parameters. In this study, the method of quantile estimators was used (Karian and Dudewicz 2011; George 2007; Slifker and Shapiro 1980).

The GEV distribution is described by a location parameter $\mu$, a scale parameter $\sigma$, and a shape parameter $k$. Parameters can be determined using similar methods as those available 339 for fitting the GLD or JSD, such as the method of moments, percentiles, or quantile estimators,

340 the latter of which was used here. The GEV resembles an extreme type distribution and is 341 often used to model the smallest or largest values in a dataset. Its PDF is given as:

$$
f_{R T}(x)=\frac{1}{\sigma} \exp \left(-\left(1+k \frac{(x-\mu)}{\sigma}\right)^{\frac{-1}{k}}\right)\left(\left(1+k \frac{(x-\mu)}{\sigma}\right)^{-1-\frac{1}{k}}\right)
$$


343 An example result of the ensemble approach implemented with the three curves above is given

344 in Figure 1, which corresponds to example problem 3 discussed below. In the figure, the three

345 individual CDFs are shown, as well as the resulting ensemble. In this problem, the ensemble

346 CDF closely resembles the GEV curve, which dominated the solution (with weights of JSD,

347 GEV, and GLD given by $w_{J S D}=0.01, w_{G E V}=0.91$, and $w_{G L D}=0.08$, respectively).

\section{Example Problems}

To illustrate the ensemble approach, several example problems are considered. These include three benchmark reliability problems and two complex engineering problems utilizing nonlinear finite element analysis. Note that the benchmark problems can be expressed algebraically and are thus not of the complexity for which an FS approach is needed, nor for which it would represent the most efficient solution method. However, these are included as their solution is readily verifiable and can provide useful information as to the range of problem characteristics for which the ensemble approach can be effective. As the purpose of the example problems is to examine the effectiveness of the ensemble approach rather than produce exact solutions, only 1000 simulations of the actual response function $R\left(X_{j}\right)$ were used to generate the dataset to fit the stand-alone CDFs, even for those problems approaching a reliability index of 4 (with corresponding failure probability of about 1:30,000). Although this produced reasonably accurate solutions for the problems considered, additional accuracy can

362 generally be obtained with additional simulations. In this study, once the ensemble CDF was

363 formed, MCS was used to quickly compute $p_{f}$ of the resulting simple two RV limit state $g^{*}=$ $364 R-Q$ (using $1 \times 10^{6}-10^{7}$ simulations, depending on reliability level), although a less expensive alternative method such as FORM would have produced solutions with no significant loss of

366 accuracy as well. Results are reported in terms of reliability index $\beta$ with the standard normal 367 transformation $\beta=-\Phi^{-1}\left(p_{f}\right)$. 
Problem Set 1: Benchmark Limit State Functions

method evaluation. Two of these cases, a multiple reliability index function and a maximum

372 function, were found by the authors of this study to be among the more difficult to solve

373 accurately with traditional analytical methods such as FORM, and are evaluated below. In each

374 of the benchmark problems, the reference solution (taken as the "exact" solution) was computed using a sample size of $1 \times 10^{9}$ with MCS.

The multiple reliability index case represents a hyperbolic function with two reliability

377 indices, and is given as:

$$
g=x_{1} x_{2}-k
$$

where $x_{1}$ (taken as control variable) and $x_{2}$ are normal RVs having mean values of 78064.4 and 0.0104, with corresponding standard deviations of 11709.7 and 0.00156 , respectively, and constant $k$ was taken as 480,240, and 160 in this study to produce different reliability levels to investigate. As shown in Table 1, although accuracy was effected when limiting to 1000 simulations, the ensemble produced superior results to the GEV and GLD distributions alone, while the JSD fit produced no failures (weights: $w_{G E V}=0.99 ; w_{J S D}$ and $w_{G L D}<0.01$ for all values of $k$ ). Note that even if a CDF was found to be ineffective by itself, such as the JSD and GLD in this case, it was still included in the ensemble. However, it was found that curves that 387 produced no failures resulted in an insignificant weight (i.e. near zero) in the ensemble, indicating, as expected, a poor fit to the data. Traditional FORM and MCS solutions are provided for comparison. As expected, MCS could produce no failures (i.e. $p_{f}=0$ ) when limiting to 1000 simulations, while two different FORM algorithms were used to solve the

391 problem (based on those of Rackwitz and Fiessler (1978) and Ayyub and Haldar (1984)), which

392 resulted in different MPPs and correspondingly different reliability indices. Here it should be 
393 pointed out that the purpose of providing the FORM and MCS comparison solutions is not to suggest that all of the many available variants of these approaches are unable provide a satisfactory solution, but rather to illustrate that the example problems are reasonably challenging and provide some difficulty for traditional approaches. several sub-functions, and results in a non-smooth limit state boundary. It is given as:

$$
g=\max \left(g_{1}, g_{2}, g_{3,} g_{4}\right)
$$

400 where:

401

402

403

404

405

406

407

408

A third analytical problem, a circular limit state function, is presented that considers non-normal random variables. In this example, random variables are considered to be either both lognormal or both extreme type I. The limit state function is given as:

$$
g=r^{2}-x_{1}^{2}-x_{2}^{2}
$$

where $r^{2}$ is taken as 7.0 for the lognormal case and 9.0 for the extreme I case. For both cases, the means and standard deviations of the random variables were taken as 1.0 and 0.25 , respectively ( $x_{1}$ taken as control variable). Results are given in Table 3, where it can be seen 
that the ensemble produced better results than the three individual distributions considered (weights: $w_{G E V}=0.99 ; w_{G L D}$ and $w_{J S D}<0.01$ for both types of RVs).

\section{Problem 2: Nonlinear Truss with Complex Random Variable Set}

This problem is based on that described in Eamon and Charumas (2011), and is meant

to represent complexity within the range of that for which the FS approach was intended. As shown in Figure 2, a 10 member truss with a non-linear material model is subjected to a load

$P$. Solution of the problem cannot be achieved with a closed-form analytical expression, and a

finite element code (ABAQUS Version 6.11-2) using 10 two-node truss elements (8 total nonzero degrees of freedom) was used to evaluate the response, as solved using the (implicit)

Newton-Raphson approach with a residual convergence criteria of 0.005 . The material assumed was steel, with a bilinear stress-strain curve and an elastic modulus $E$ of $200 \mathrm{GPa}$. Random variables are the cross-sectional area $(A)$, yield stress $\left(\sigma_{y}\right)$, and post-yield modulus $\left(E_{2}\right)$ of each truss member, and load $(P)$ (taken as control variable). Random variables are taken to have different types of distribution, level of variance, and correlation, as summarized in Table 4.

Note that for the normal RVs $A$ and $E_{2}$, negative values are theoretically possible during the simulation, potentially producing a physically impossible problem as well as a failed FEA solution attempt. Since only 1000 simulations were used to generate the data sample for $R\left(\boldsymbol{X}_{j}\right)$, this did not occur (and represents an improbable result, as negative values occur at 10 and 20 standard deviations from the means of $A$ and $E_{2}$, respectively). However, for cases in which this would be a concern, alternative distributions or appropriate truncated RV types could be used.

441 The failure criterion was defined as the state where the stress in member 1 reaches its yield stress. The resulting limit state function is given as:

$$
g=\sigma_{y l}-\sigma_{l}\left(P, \sigma_{y j}, E_{2 i}, A_{i}\right) \text { for } i=1 \text { to } 10, j=2 \text { to } 10
$$


444 The reference solution $(\beta=3.50)$ was obtained from $1 \times 10^{6}$ MCS simulations. Note that

445 although the limit state function was evaluated with a sample size of 1000 , (the "nominal"

446 number of calls), the actual number of function calls using the ensemble approach exceeded

447 this value, due to the iterative process needed to find the root of $g^{*}=0$, as shown in Table 5;

448 such iteration is not required for the explicitly formulated response functions in problem set 1 ,

449 for which roots can be determined analytically. Here a version of the bracketing method was

450 used for solution (Suhadolnik 2012), with an error tolerance of 2\%. For comparison, and to

451 verify the suitability of problem complexity, a FORM solution was also attempted, and failed

452 to provide a solution, as the MPP could not be located, even after using several different search

453 algorithms and different starting points. Similarly, as expected, no solution could be obtained

454 from MCS when limiting the actual number of function calls to that of the ensemble approach.

455 In this problem, the GEV dominated the solution (weights: $w_{G E V}=0.753 ; w_{G L D}=0.0011 ; w_{J S D}$

$456=0.246$ ), and thus the simplified threshold method was used for illustration in lieu of the

457 complete ensemble, from which good results were obtained.

458

459 Problem 3: Highly Nonlinear Column with Large Random Variable Set

460

This problem is based on that given by Alsendi and Eamon (2020). It represents a

reinforced concrete bridge pier column subjected to a blast load initiated at the column base.

462 The column base is fixed and the top is constrained by a beam element representing the pier

463 beam cap, which is connected to two additional columns forming the pier structure, which are

464 also modeled with beam elements (not shown in Figure 3 for clarity). The column is $3 \mathrm{~m}$ high and $760 \mathrm{~mm}$ square, and reinforced with 24 \#8 vertical bars (6 bars per face) and \#4 ties spaced at $300 \mathrm{~mm}$. An axial load is applied to the column representing the dead load portion of a two-

467 lane, two-span (15 m per span) continuous bridge with a superstructure of five steel girders

468 (spaced at $2.7 \mathrm{~m}$ ) and a $240 \mathrm{~mm}$ thick reinforced concrete deck that the column supports. 
Resistance random variables are concrete compressive strength $\left(f^{\prime}{ }_{c}\right)$, yield stress $\left(F_{y l}\right.$; $\left.F_{y t}\right)$, Young's modulus $\left(E_{l} ; E_{t}\right)$, and tangent modulus $\left(E_{T l} ; E_{T t}\right)$ of the longitudinal bars $(l)$ and ties $(t)$. Random variables associated with each longitudinal bar are taken as independent of each other, while those for transverse bars are taken as perfectly correlated (for random variables of the same type), resulting in 75 total random variables characterizing steel uncertainties, as summarized in Table 6. Load random variables are those of the bridge gravity load and blast load. Gravity (dead) load random variables are those of the prefabricated items such as the steel girders and diaphragms $\left(D L_{p}\right)$; the cast-in place items such as the deck and barriers $\left(D L_{s}\right)$; and the wearing surface $\left(D L_{w}\right)$. Statistical parameters for concrete and steel yield strength are taken from Nowak and Szerszen (2003), while statistics for steel stiffness are taken from Galambos and Ravindra (1978), and those for gravity loads are taken from Nowak (1999). The blast load random variables are the equivalent mass of TNT $(\mathrm{kg})\left(Q_{w}\right)$ and the net equivalency factor $\left(Q_{e}\right)$ (taken as control variable), where variation in $Q_{w}$ is meant to account uncertainty in charge weight construction and $Q_{e}$ accounts for uncertainty in the resulting blast pressure. Statistical parameters for these two random variables are taken from Netherton and Stewart (2010). All distributions are reported to be normal except $Q_{e}$, which is triangular. In total, 81 random variables were considered.

In this problem, failure is defined as a horizontal displacement of the column base that exceeds $4.3 \mathrm{~mm}$ within the first $8 \mathrm{~ms}$ after the blast initiates (a rate of deformation associated with subsequent column collapse). The resulting limit state function is given as:

$$
g=4.3-D(\boldsymbol{R}, \boldsymbol{Q})
$$

where $D$ is the maximum displacement of the column base resulting from the blast at a time of $4918 \mathrm{~ms}$, and $\boldsymbol{R}$ and $\boldsymbol{Q}$ are the sets of resistance and load random variables given in Table 6 . 492 Response $D$ was evaluated using a large strain, large displacement FEA approach, where the 493 model had approximately 4800 8-node hexahedral (for concrete) and 2-node beam (for 
494 reinforcement) elements. Concrete was represented with the Holmquist-Cook model

495 (Holmquist et al. 1993), which accounts for crushing and cracking due to accumulated damage

496

497

498

499

500

501

502

503

504

505

506

507

508

509

510

511

512

513

514

515

516

517

518

under high rates of strain. Reinforcing steel is modeled with a kinematic, bi-linear material model, while the blast load time-pressure history was represented by the CONWEP method (Hyde 1988). The problem was solved on 4 CPUs in parallel using the finite element code LSDYNA. As this problem is significantly nonlinear, with the displacement response $D$ fairly sensitive to parameter changes, as shown in Table 7, about twice the number of iterations were required to determine the root of the limit state function than for the nonlinear truss problem previously studied. Consideration of a more sophisticated root finding algorithm than the bracketing method used may further reduce this requirement, however. As shown in Table 7, the ensemble provided best results overall (weights: $w_{G E V}=0.91 ; w_{G L D}=0.08 ; w_{J S D}=0.01$ ), whereas GLD could not be fit to the resistance data, JSD produced a relatively high error, and FORM could not converge to a solution.

\section{Conclusion and Recommendations}

Previous formulations of the failure sampling method were limited by uncertainty with the method of implementation, where the approach with greatest accuracy is highly problemspecific. In this study, a solution was proposed to this issue that reduces this uncertainty and increases the effectiveness of the method regardless of the problem considered. It was found that the ensemble approach is suitable for complex responses and highly nonlinear limit state boundaries. It was further found that the approach is expected to produce solutions at least as good, and often better, than the best single failure sampling implementation method previously presented in the literature. Although the ensemble method is thus recommended for implementation of the failure sampling approach, significant opportunities exist for further development. Among these, more rigorously exploring alternative goodness-of-fit metrics, 
519 and formulating the ensemble using a different approach, are the most apparent to the authors.

520 For example, rather than first fitting individual CDFs to the response data then finding the 521 associated weights, perhaps a more universal optimization could be conducted where the 522 individual curve parameters as well as the curve weights are taken as a single set of design 523 variables in the same optimization process. As all curve parameters are thus interrelated, the 524 end result, a single unified curve, may offer greater ability to represent the response data than 525 the weighted independently developed curves. Such topics are to be further explored in the 526 future.

$530 \quad$ Data Availability

531 Some or all data, models, or code that support the findings of this study are available

533 from the corresponding author upon reasonable request. 


\section{References}

Acar E and Rais-Rohani M. (2009). "Ensemble of Metamodels with Optimized Weight Factors.” Structural and Multidisciplinary Optimization. Vol. 37, No. 3, p. 279-294.

Acar E Rais-Rohani M, and Eamon C. (2010). "Reliability Estimation Using Univariate Dimension Reduction and Extended Generalized Lambda Distribution.” International Journal of Reliability and Safety, Vol. 4, No. 2/3, p. 166-187.

Alsendi A and Eamon, C. (2020). "Quantitative Resistance Assessment of SFRP-Strengthened RC Bridge Columns Subjected to Blast Loads.” Journal of Performance of Constructed Facilities, Vol. 34, No. 4.

Ang A and Tang W. (2007). Probability Concepts in Engineering: Emphasis on Applications to Civil and Environmental Engineering. Wiley: New York.

Asif L and Helmut M. (2000). "Estimating The Parameters Of The Generalized Lambda Distribution." ALGO Research Quarterly, Vol. 3, p. 47-58.

Au S and Beck J. (2001). "Estimation Of Small Failure Probabilities In High Dimensions By

Au S, Ching J, and Beck J. (2007). "Application Of Subset Simulation Methods To Reliability Benchmark Problems." Structural Safety, Vol. 29, p. 183-193.

Ayyub B and Chia C. (1992). "Generalized Conditional Expectation For Structural Reliability Assessment." Structural Safety, Vol. 11, p. 131-146.

Ayyub B and Haldar A. (1984). "Practical Structural Reliability Techniques." ASCE Journal of Structural Engineering, Vol. 110, p. 1707-1724.

Breitung K. (1984) "Asymptotic Approximations for Multinormal Integrals." ASCE Journal of Engineering Mechanics, Vol. 110, p. 357-366. 
Cheng J and Li Q. (2009). "Application Of Response Surface Methods To Solve Inverse Reliability Problems With Implicit Response Functions." Computational Mechanics, Vol. 43. p. 451-459.

Chiralaksanakul A, and Mahadevan S. (2005). "First Order Methods For Reliability Based Optimization." Journal of Mechanical Design, Vol. 127, p. 851-857.

Ditlevesen P and Bjerager P. (1988). "Plastic Reliability Analysis By Directional Simulation." ASCE Journal of Engineering Mechanics, Vol. 115, p. 1347-62.

Eamon C, and Charumas B. (2011). "Reliability Estimation Of Complex Numerical Problems Using Modified Conditional Expectation Method." Computers and Structures, Vol. 89 , p. $181-188$.

Eamon C, Thompson M, and Liu Z. (2005). "Evaluation Of Accuracy And Efficiency Of Some Simulation And Sampling Methods In Structural Reliability Analysis." Structural Safety, Vol. 27, p. 356-392.

Engelund S and Rackwitz R. (1993). "A Benchmark Study On Importance Sampling Techniques In Structural Reliability." Structural Safety, Vol. 12. p. 255-276.

Galambos T and Ravindra M. (1978). Properties of Steel for Use in LRFD. ASCE Journal of the Structural Division, Vol. ST9, p. 1459-1468.

George F. (2007). "Johnsons System of Distribution and Microarray Data Analysis." PhD Dissertation, Department of Mathematics, University of South Florida.

Goel T, Haftka R, Shyy W, and Queipo N. (2007). "Ensemble of Surrogates.” Journal of Structural and Multidisciplinary Optimization, Vol. 33, No. 3, pp. 199-216.

Gomes H and Awruch A (2004). "Comparison Of Response Surface And Neural Network With Other Methods For Structural Reliability Analysis." Structural Safety, Vol. 26, p. 49-67. 
Gomes W (2019). "Structural Reliability Analysis Using Adaptive Artificial Neural Networks." Journal of Risk and Uncertainty in Engineering Systems Part B: Mechanical Engineering.” Vol. 5, p. 1-8.

Grooteman F. (2011). “An Adaptive Directional Importance Sampling Method for Structural Reliability.” Probabilistic Engineering Mechanics, No. 26, p. 134-141.

Guo Q, Liu Y, Chen B, and Zhao, Y. “An Active Learning Kriging Model Combined with Probabilistic Engineering Mechanics, No. 60, 103054.

Haldar A and Mahadevan S. (2000). "Probability, Reliability And Statistical Methods In Engineering Design." $1^{\text {st }}$ ed. New York: John Wiley and Sons.

Holmquist T, Johnson G, and Cook W. (1993). "A Computational Constitutive Model for 592 Concrete Subjected to Large Strains, High Strain Rates, and High Pressures.” Vol. 2, Proceedings of the 14th International Symposium of Warhead Mechanisms, Terminal Ballistics, Quebec, Canada, p. 591-600.

Hyde D (1988). User's Guide for Microcomputer Program CONWEP, Applications of TM 5855-1. Fundamentals of Protective Design for Conventional Weapons. SL-88-1. Vicksburg, MS: US Army Corps of Engineers Waterways Experiment Station Instruction.

Iman R and Conover W. (1982). "A Distribution-Free Approach To Inducing Rank Correlation Among Input Variables". Communications in Statistics, Vol. 11. p. 311334.

Karamchandani A, Bjerager P, and Cornell AC. (1989). "Adaptive Importance Sampling." 603 Proceedings, International Conference on Structural Safety and Reliability, San 604 Francisco, CA., p. 855-862. 
Karian Z and Dudewicz E. (2011). "Handbook Of Fitting Statistical Distributions With R." CRC Press.

Melchers R. (1999). "Structural Reliability Analysis and Prediction." 2nd ed. New York: John Wiley \& Sons.

Netherton M and Stewart M. (2010). "Blast Load Variability and Accuracy of Blast Load Prediction Models.” International Journal of Protective Structures, Vol 1., No. 4, p. 543-570.

Nowak A. (1999). "Calibration of LRFD bridge design code." NCHRP Report 368. Washington, DC: Transportation Research Board.

Nowak A and Collins K. (2013). "Reliability of Structures, $2^{\text {nd }}$ Ed." CRC Press, New York.

Nowak A and Szerszen M. (2003). "Calibration of Design Code for Buildings (ACI 318); Part 1 - Statistical Models for Resistance.” ACI Structural Journal, No. 100, p 377382.

Ozaturk A and Dale R. (1985). "Least Square Estimation Of The Parameters Of The Generalized Lambda Distribution." Technometrics, Vol. 27, p. 81-84.

Patki K and Eamon C. (2016). "Evaluation of Alternative Implementation Methods of a Failure Sampling Approach for Structural Reliability Analysis.” ASCE Journal of

Patki, K., and Eamon, C. (2014). “Application of MCMC in Failure Sampling." in Vulnerability, Uncertainty, and Risk: Quantification, Mitigation, and Management Proceedings of the 2nd International Conference on Vulnerability and Risk Analysis and Management and the 6th International Symposium on Uncertainty Modeling and Analysis, American Society of Civil Engineers p. 2125-2136. 
630 Rackwitz R, and Fiessler B. (1978). "Structural Reliability Under Combined Random Load $631 \quad$ Sequences." Computers and Structures, Vol. 9, p. 484-494.

632 Rubinstein R. (1981). "Simulation And The Monte Carlo Method." $1^{\text {st }}$ ed. New York: John $633 \quad$ Wiley \& Sons.

634 Shayanfar, M., Barkhordari, M., Barkhori, M., and Barkhori, M. (2018). "An Adaptive 635 Directional Importance Sampling Method for Structural Reliability Analysis." 636 Structural Safety, No. 20, p. 14-20.

637 Slifker J, and Shapiro S. (1980). "The Johnson System: Selection And Parameter 638 Estimation." Technometrics, Vol. 22, p. 239-246.

639 Suhadolnik, A. (2012) "Combined Bracketing Methods for Solving Nonlinear Equations.” 640 Applied Mathematics Letters, Vol. 25, p. 1755-1760.

641 Wu Y. (1992). "An Adaptive Importance Sampling Method For Structural Systems 642 Analysis, Reliability Technology." ASME Winter Annual Meeting, AD 28, p. 217643 231.

644 Zerpa L, Queipo N, Pintos S, and Salager J. (2005). “An Optimization Methodology of 645 Alkaline-Surfactant-Polymer Flooding Processes Using Field Scale Numerical 646 Simulation and Multiple Surrogates.” Journal of Petroleum Science and Engineering, 647 No. 47 , p. 197-208.

648 
649 Table 1. Hyperbolic Function Results.

\begin{tabular}{lccccccc}
\hline \multirow{2}{*}{ method } & no. of & \multicolumn{2}{c}{$\mathrm{k}=480$} & \multicolumn{2}{c}{$\mathrm{k}=240$} & \multicolumn{2}{c}{$\mathrm{k}=160$} \\
& calls & $\beta$ & $\%$ err & $\beta^{*}$ & $\%$ err & $\beta^{*}$ & $\%$ err \\
\hline Reference solution (MCS) & $1 \times 10^{9}$ & 2.10 & -- & 4.15 & -- & 4.98 & -- \\
FORM $* *$ & $8-40$ & $2.18 ; 2.22$ & $3.8 ; 5.7$ & $4.32 ; 4.41$ & $3.1 ; 6.2$ & $5.19 ; 5.21$ & $4.2 ; 4.6$ \\
MCS & 1000 & 2.10 & 0.0 & NF & -- & NF & -- \\
\hline GLD & 1000 & 1.48 & 30 & 3.71 & 10.6 & 3.92 & 21 \\
JSD & 1000 & 2.75 & 31 & NF & -- & 5.27 & 5.8 \\
GEV & 1000 & 2.11 & 0.48 & 4.31 & 3.9 & 5.22 & 4.8 \\
Ensemble & 1000 & 2.11 & 0.48 & 4.24 & 2.1 & $5.18^{* * *}$ & 4.0 \\
\hline
\end{tabular}

$650 * \mathrm{NF}=$ no failures. $* *$ Results given for alternate algorithms; see text. For FORM, no. of call depends on

651 problem and algorithm; range is given. ***Increasing the number of simulations to 2000 produced $\beta=4.96$

652 (0.4\% error).

653

654 Table 2. Maximum Function Results.

\begin{tabular}{lccc}
\hline \multicolumn{1}{c}{ method } & $\begin{array}{c}\text { no. of } \\
\text { calls }\end{array}$ & $\beta$ & \%err \\
\hline Reference solution (MCS) & $1 \times 10^{9}$ & 3.53 & -- \\
FORM & -- & Fail* & -- \\
MCS & 1000 & NF & -- \\
\hline GLD & 1000 & Fail** & -- \\
JSD & 1000 & 3.46 & 1.98 \\
GEV & 1000 & Fail** & -- \\
Ensemble & 1000 & 3.46 & 1.98 \\
\hline
\end{tabular}

655

*Solution could not converge. **Could not fit the resistance data.

656

657

658

Table 3. Circular Limit State Results.

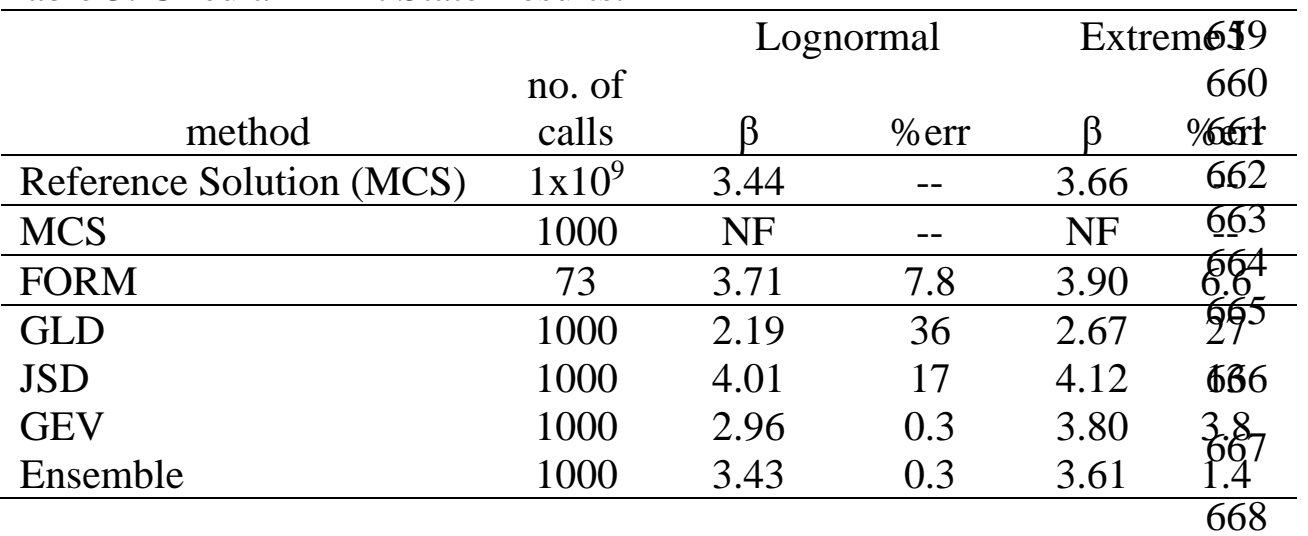

669

670 Table 4. Random Variables for Truss Problem.

\begin{tabular}{lccccl}
\hline Random Variable & Total & Mean & COV $^{*}$ & $\rho^{* *}$ & Distribution \\
\hline area $(A)$ & 10 & $1290 \mathrm{~mm}^{2}$ & 0.05 & 0.30 & normal \\
yield stress $\left(\sigma_{y}\right)$ & 10 & $345 \mathrm{MPa}$ & 0.15 & 0.50 & lognormal \\
post-yield modulus $\left(E_{2}\right)$ & 10 & $8280 \mathrm{MPa}$ & 0.10 & 0.70 & normal \\
load $(P)$ & 1 & $85 \mathrm{kN}$ & 0.35 & -- & extreme I \\
\hline
\end{tabular}

671

*Coefficient of variation.

672

**Correlation coefficient between random variables of the same type between different truss members.

673

674 
677 Table 5. Truss Problem Results.

\begin{tabular}{lcccc}
\multicolumn{1}{c}{ method } & $\begin{array}{c}\text { nominal } \\
\text { no. of } \\
\text { calls }\end{array}$ & $\begin{array}{c}\text { actual } \\
\text { no. of } \\
\text { calls }\end{array}$ & $\beta$ & \%err \\
\hline Reference solution (MCS) & $1 \times 10^{6}$ & $1 \times 10^{6}$ & 3.50 & -- \\
FORM & -- & -- & Fail & -- \\
MCS & 3000 & 3000 & NF & -- \\
\hline GLD & 1000 & 3000 & 3.01 & 14 \\
JSD & 1000 & 3000 & 4.46 & 27 \\
GEV & 1000 & 3000 & 3.48 & 0.57 \\
Ensemble & 1000 & 3000 & 3.48 & 0.57 \\
\hline
\end{tabular}

678

679

680

Table 6. Random Variables for Column Problem.

\begin{tabular}{|c|c|c|c|c|}
\hline Random Variable (RV) & Total & Nominal value & Bias factor* & $\mathrm{COV}^{* *}$ \\
\hline \multicolumn{5}{|l|}{ Resistance RVs } \\
\hline Concrete strength $\left(f_{c}^{\prime}\right)$ & 1 & $41 \mathrm{MPa}$ & 1.15 & 0.15 \\
\hline Yield stress, long. bars $\left(F_{y l}\right)$ & 24 & $414 \mathrm{MPa}$ & 1.14 & 0.05 \\
\hline Yield stress, ties $\left(F_{y t}\right)$ & 1 & $276 \mathrm{MPa}$ & 1.145 & 0.05 \\
\hline Young's Modulus, long. bars $\left(E_{l}\right)$ & 24 & $200 \mathrm{GPa}$ & 1.0 & 0.04 \\
\hline Young's Modulus, ties $\left(E_{t}\right)$ & 1 & $200 \mathrm{GPa}$ & 1.0 & 0.04 \\
\hline Tangent modulus, long. bars $\left(E_{T l}\right)$ & 24 & $20 \mathrm{GPa}$ & 1.0 & 0.04 \\
\hline Tangent modulus, ties $\left(E_{T t}\right)$ & 1 & $20 \mathrm{GPa}$ & 1.0 & 0.04 \\
\hline \multicolumn{5}{|l|}{ Load RVs } \\
\hline Weight, prefab items $\left(D L_{p}\right)$ & 1 & $67 \mathrm{kN}$ & 1.03 & 0.08 \\
\hline Weight, cast in place items $\left(D L_{s}\right)$ & 1 & $387 \mathrm{kN}$ & 1.05 & 0.10 \\
\hline Weight, wearing surface $\left(D L_{w}\right)$ & 1 & $134 \mathrm{kN}$ & Mean $=89 \mathrm{~mm}$ & 0.25 \\
\hline Charge weight $\left(Q_{w}\right)$ & 1 & $47 \mathrm{~kg}$ & 1.000 & 0.10 \\
\hline Equivalency factor $\left(Q_{e}\right)$ & 1 & 1.00 & Mode $=0.82$ & 0.36 \\
\hline
\end{tabular}

681

*Ratio of mean to nominal value. **Coefficient of variation. Not available for $D L_{w}$ and $Q_{e}$ as shown.

682

683

684

Table 7. Column Problem Results.

685

\begin{tabular}{lcccc}
\hline \multicolumn{1}{c}{ method } & $\begin{array}{c}\text { nominal } \\
\text { no. of } \\
\text { calls }\end{array}$ & $\begin{array}{c}\text { actual } \\
\text { no. of } \\
\text { calls }\end{array}$ & $\beta$ & \%err \\
\hline Reference solution (MCS) & $1 \times 10^{6}$ & $1 \times 10^{6}$ & 3.89 & -- \\
FORM & -- & -- & Fail & -- \\
MCS & 6000 & 6000 & NF & -- \\
\hline GLD & 1000 & 6000 & Fail & -- \\
JSD & 1000 & 6000 & 3.40 & 13 \\
GEV & 1000 & 6000 & 4.01 & 3.1 \\
Ensemble & 1000 & 6000 & 3.80 & 2.3 \\
\hline
\end{tabular}




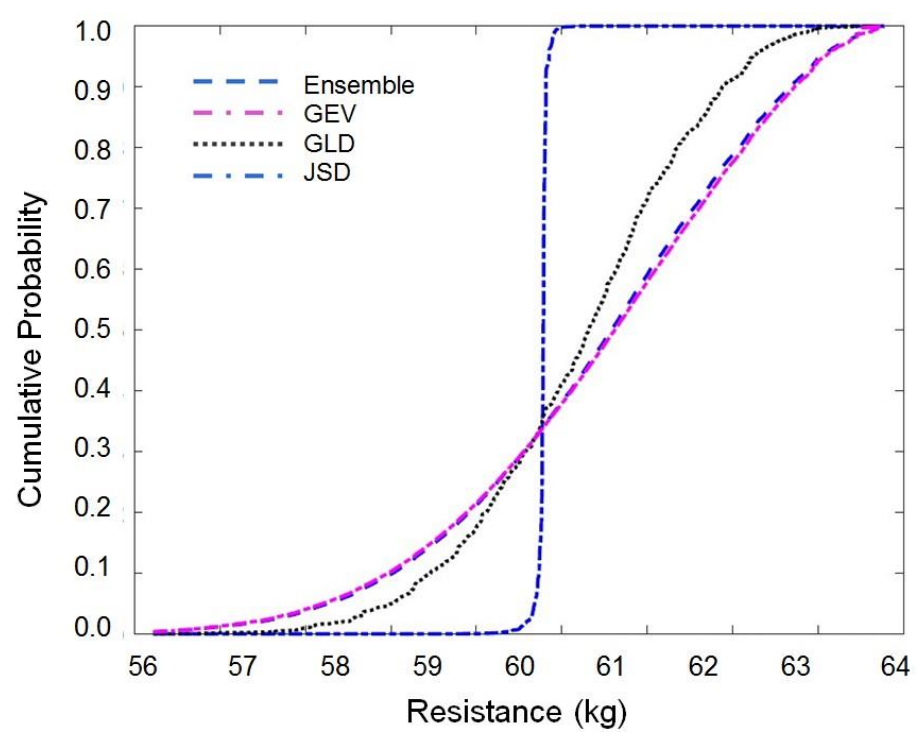

687

688

689 Figure 1. Example Ensemble of CDFs .

690

691

692

693

694

695

696

697

698

699

700

701

702

703

704

705

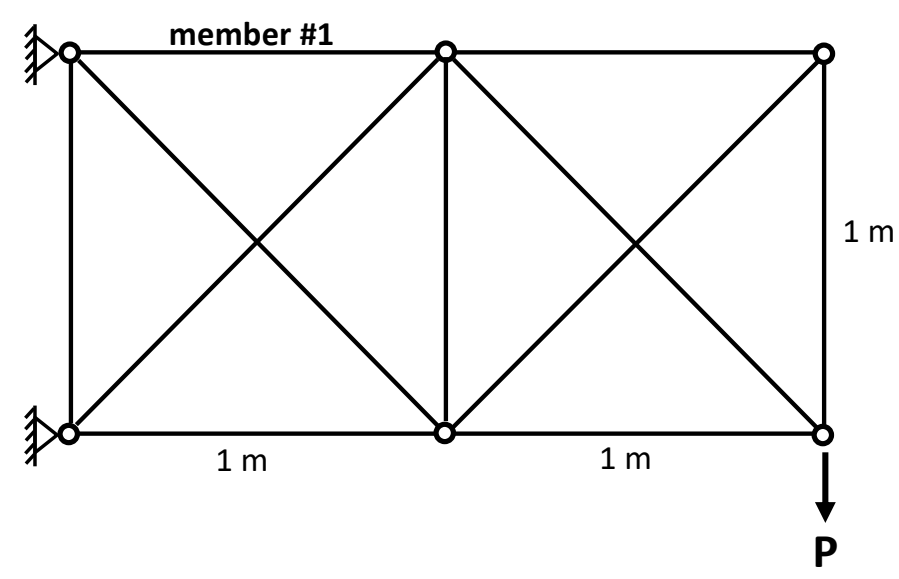

Figure 2. Ten Bar Truss.

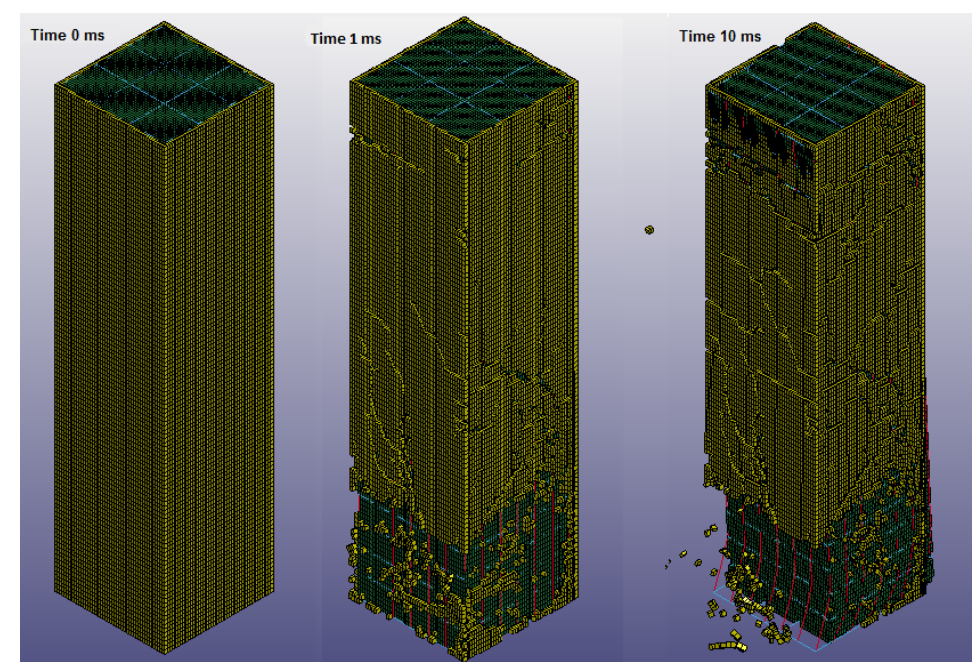

708 Figure 3. Column Subjected to Blast Load. 